\title{
Validación de la versión española del inventario de asertividad Gambrill y Richey en población con diagnóstico de esquizofrenia
}

\author{
Emma Casas-Anguera ${ }^{1 *}$, Gemma Prat ${ }^{2}$, Sonia Vilamala ${ }^{1}$, Maria J. Escandell ${ }^{1}$, Mar Garcia-Franco ${ }^{1}$, \\ José R. Martin ${ }^{1}$, Ester López ${ }^{2}$ y Susana Ochoa ${ }^{1}$ \\ ${ }^{1}$ Parc Sanitari Sant Joan de Déu, Sant Boi de Llobregat. Spain. CIBERS AM \\ ${ }^{2}$ Divisió de Salut Mental de la Fundació Althaia, Manresa. Spain.
}

\begin{abstract}
Resumen: Objetivo: Validar la versión española del Cuestionario de Asertividad de Gambrill y Richey en personas con esquizofrenia. Método: La muestra está compuesta por 125 personas con esquizofrenia en tratamiento en uno de los centros de rehabilitación psicosocial que participan en el estudio. Los instrumentos utilizados para valorar la validez discriminante y convergente son:el cuestionario de habilidades de comunicación(CSQ); la Escala de Funcionamiento Social(EFS); el Perfil de Habilidades de la Vida Cotidiana(LSP);la Escala de Impresión Clínica Global(ICG) y la Escala de Funcionamiento Global(GAF). Resultados: La consistencia interna del instrumento muestra un alfa de Cronbach de .935 en la escala total. Los coeficientes de correlación intraclase para valorar la consistencia temporal del instrumento oscilan entre .21 y .78. El instrumento muestra validez convergente con la escala CSQ y con las subescalas de comunicación y aislamiento social $(p<.001-.05)$. Con el resto de subescalas de funcionamiento social muestra validez divergente. Con la CGI muestra validez divergente con la probabilidad de respuesta, sin embargo encontramos que hay validez convergente entre las subescalas de CGI y la subescala de grado de malestar del instrumento $(p<.012-.042)$. Conclusiones: El instrumento muestra unas buenas características psicométricas y parece útil para la valoración de las habilidades sociales en una muestra de personas con esquizofrenia.

Palabras Clave: Esquizofrenia; rehabilitación; asertividad; habilidades sociales.
\end{abstract}

\section{Introducción}

Los déficits en las habilidades sociales son una de las principales características descriptivas de las personas con esquizofrenia y se consideran como centrales en el denominado déficit del funcionamiento social o de la falta de competencia social (Green, Kem, Braff y Mintz, 2000; Heinssen, Liberman y Kopelowicz, 2000; Liberman y Kopelowicz, 2004). Este déficit se relaciona con una disminución de la calidad de vida y es un factor predictivo de evolución clínica desfavorable, recaídas y desempleo (Otero 2004; Wallace y Liberman, 1985). Por ello, el fomento de la competencia social mediante el entrenamiento en habilidades sociales es uno de los aspectos que se ha relacionado con el atenuamiento y la compensación de los efectos nocivos de los déficits cognitivos, la vulnerabilidad, los acontecimientos estresantes y el desajuste social; previniendo la recaída, mejorando la resistencia y la calidad de vida, al mismo tiempo favoreciendo las relaciones interpersonales de los pacientes con esquizofrenia (Anthony, Cohen y Cohen, 1984; Caballo, 1993; Farkas y Anthony, 1982).

En este sentido, se han diseñado diferentes programas de intervención encaminados a mejorar los déficits de funcionamiento social (Farkas, Anthony y Cohen, 1989; Labrador,

* Dirección para correspondencia [Correspondence address]: Emma Casas-Anguera. Parc Sanitari Sant Joan de Déu. C/ Doctor Antoni Pujades, 42. 08830 Sant Boi de Llobregat. Barcelona (España). E-mail: ecasas@pssjd.org
Title: Validation of the Spanish version of the inventory of Gambrill and Richey assertiveness in people diagnosed with schizophrenia.

Abstract: Objective: To validate the Spanish version of the questionnaire Gambrill and Richey Assertiveness for people with schizophrenia. Method: he sample comprised a total of 125 people with schizophrenia on treatment in one of the psychosocial rehabilitation centers participating in the study. The instruments used to assess convergent and discriminant validity are the Communication Skills Questionnaire(CSQ); the Social Functioning Scale(SFS); the Life Skills Profile(LSP); the Clinical Global Impression Scale(CGI) and the Global Functioning Scale (GAF). Results: Internal consistency of the instrument shows a Cronbach alpha of .935 in the full scale. The intraclass correlation coefficients to assess the instrument's internal consistency ranged between .21 and .78. The instrument shows a convergent validity with CSQ scale and the subscales of communication and social isolation $(p<.001-.05)$. With the rest of social functioning subscales shows divergent validity. With the CGI shows divergent validity with the probability of response, and convergent validity between the subscales of CGI and the degree of distress subscale of the instrument $(p<.012$ .042). Conclusions: The instrument shows good psychometric characteristics and appears useful for assessing social skills in a sample of people with schizophrenia.

Key words: Schizophrenia; rehabilitation; assertiveness; social skills.

2004; Liberman, 1995; Liberman, Wallace, Blackwell, Eckman, Baccaro y Kuenhel, 1993; Orviz y Fernández, 2002). No obstante, el progreso en el desarrollo de intervenciones efectivas se ha enlentecido debido a la ausencia de medidas válidas y fiables para ser utilizadas, de forma que las distintas mediciones no se realizan de forma adecuada, limitando la evaluación de su eficacia y efectividad.

Un aspecto crítico global de las habilidades sociales es la conducta asertiva, que se puede definir como la que afirma, asegura y ratifica las opiniones propias en situaciones interpersonales y tiene como consecuencia producir y mantener autorefuerzos sin utilizar conductas agresivas hacia los otros. Es decir, presenta cuatro componentes característicos: a) afirmación de opiniones personales, b) situación interpersonal, c)consecución de autorefuerzos, d) no utilización de conductas agresivas. La valoración de la conducta asertiva se realiza a menudo mediante técnicas de Role playing a partir de conversaciones simuladas. Este tipo de valoración es muy poco operativa de manera que se dificulta la medición de estas capacidades. Asimismo, se encuentra influenciada por factores subjetivos, hecho que dificulta la comparación de datos.

Por todo ello, es necesario obtener medidas válidas y fiables de la conducta asertiva para pacientes con esquizofrenia. Uno de los cuestionarios más utilizados en el ámbito clínico ha sido el Inventario de Asertividad de Gambrill y Richey (Gambrill y Richey, 1975). En la actualidad, en nuestro ámbito geográfico este instrumento ha sido validado para su uso 
en la población general (Carrasco, Clemente y Llavona, 1989; Raich, Escursell y Vila-Vidal, 1985), pero no para su utilización en población con un diagnóstico de esquizofrenia. Ante esta situación se hace necesario la adaptación y validación de este instrumento en población con esquizofrenia. Por lo tanto, el objetivo del presente trabajo consiste en realizar la validación de la versión española del Cuestionario de Asertividad de Gambrill y Richey en personas con esquizofrenia.

\section{Método}

\section{Participantes}

La muestra está compuesta por un total de 125 personas con diagnóstico de trastorno esquizofrénico según criterios del DSM-IV-TR, entre 18 y 65 años que residen en la provincia de Barcelona (65 procedentes de diferentes servicios de rehabilitación comunitaria, servicio prelaboral y del Servicio Especializado en Rehabilitación Psiquiátrica Intensiva de la Unidad Hospitalaria de Larga Estancia del Parc Sanitari Sant Joan de Déu y 60 de la Divisió de Salut Mental de la Fundació Althaia). El Parc Sanitari Sant Joan de Déu cubre un área de atención de 1.199.389 (a fecha de 2007) de la población general; la Fundació Althaia cubre una población de 215.000 personas. No se incluyeron aquellos que presentaban un trastorno mental orgánico o un diagnóstico comórbido de retraso mental, descompensación clínica que requiera ingreso en unidades de hospitalización de agudos o expresaran el deseo de no participar en el estudio.

\section{Instrumentos}

- Datos sociodemográficos en el que se recogían datos como la edad, fecha de nacimiento, género, estado civil, estudios realizados, núcleo de convivencia y situación laboral.

- Inventario de Asertividad (Gambrill y Richey, 1975). Este inventario autoaplicado valora la asertividad en población general. Hasta el momento es el instrumento que frecuentemente se utiliza para la valoración de las habilidades sociales en programas de entrenamiento en esta área. Es un instrumento que está compuesto de 40 ítems que valoran en dos subescalas el grado de malestar y la probabilidad de emisión de respuesta ante una determinada situación social, a través de una escala tipo Likert de 5 puntos. Se obtiene una puntuación general de asertividad con las puntuaciones de las dos subescalas. Una alta puntuación indicaría una disfunción, mientras que puntuaciones bajas indicarían pocas dificultades.

- Cuestionario de habilidades de comunicación CSQ (Takahashi, Tanaka y Miyaoka, 2006) que consta de 29 ítems. Se trata de un cuestionario autoadministrado. Los ítems se distribuyen en dos factores que valoran habilidades generales de comunicación y habilidades interpersonales de comunicación. Los 29 ítems de este cuestionario se agrupan en dos bloques: uno primero de 6 ítems (puntuación de 0- deficiente-5-siempre buena, tipo Likert) que valora habilidades generales de comunicación y uno segundo de 29 ítems (puntuación de 0 - deficiente- 2-buena la mayoría de veces, tipo Likert) que valora habilidades interpersonales de comunicación en 6 ámbitos diferentes: familia, amigos íntimos, amigos, vecinos, figuras de autoridad y extraños. Se ha realizado la adaptación y validación en muestra española (Prat, Casas-Anguera, Garcia-Franco, Escandell, Martín, Vilamala, Villalta-Gil, Gimenez-Salinas, HernandezRambla y Ochoa, 2009).

- Escala de funcionamiento social (SFS) (Birchwood, Smith, Cochrane, Wetton y Copestake, 1990). Esta escala permite valorar el funcionamiento social en diferentes áreas y está dirigido a personas con diagnóstico de esquizofrenia, utilizándose ampliamente para la planificación del proceso de rehabilitación (Birchwood et al., 1990). Las áreas que incluye son: Aislamiento social (AS), Comunicación interpersonal (CINT); Independencia-ejecución (IE); Independenciacompetencia (IC); Actividades de tiempo libre (ATP); Conducta prosocial (CP); Empleo-ocupación (EO). Se ha utilizado la versión heteroaplicada de Olivares y Torres (2005).

- Escala sobre Perfil de habilidades de la vida cotidiana (LSP): Se trata de una escala de 39 ítems que se agrupan en 5 factores: autocuidado (10 a 40), comportamiento socialinterpersonal (10 a 40), comunicación-contacto social (6 a 24), comportamiento social no personal (6 a 24) y vida autónoma (7 a 28). Cada ítem es valorado según cuatro posibilidades, de 1 a 4, aumentando en función de la incapacidad (Rosen, Hadzi-Paulovic y Porter, 1989). La escala evalúa aspectos de funcionamiento que afectan en las actividades diarias y en la adaptación de las personas con enfermedades psiquiátricas a la comunidad, valorando tanto capacidades como déficits. A una mayor puntuación en cada factor mayor capacidad en esa área. Las subescalas centradas en valorar el funcionamiento social (comportamiento social inter-personal; comportamiento social no-personal y comunicación y contacto social) de la persona (Fernández de Larrinoa, Bulbena y Domínguez, 1992) se han utilizado para la validez concurrente, mientras que el resto de escalas se han utilizado para la validez divergente. Se ha utilizado la versión castellana validada por Bulbena, Burgés, Fernández, Autonell y Melloni (2007), ampliamente utilizada para diseñar los programas de rehabilitación de los pacientes con trastorno mental severo

- Escala de Funcionamiento Global (GAF) (Endicott, Spitzer, Fleiss y Cohen, 1976). Mide el funcionamiento general del paciente en una escala que oscila entre 0 y 100 , obteniendo mayores puntuaciones aquellas personas que tienen un mejor funcionamiento. Es una escala ampliamente utilizada ya que proporciona información general de los pacientes y la evaluación es breve.

- Escala de Impresión Clínica Global (ICG) (Haro, Kamath, Ochoa, Novick, Rele, Fargas, Rodriguez, Rele, Orta, Kharbeng, Araya, Gervin, Alonso, Maureas, Lavrentzou, Liontos, Grefor, Jones, SOHO Study Group, 2003). Valora la psicopatológica que presenta el sujeto en cuatro 
subescalas: síntomas positivos, negativos, cognitivos, depresivos y una puntuación global de psicopatología.

Los pacientes fueron evaluados en dos momentos: evaluación basal y a la semana. Para la validez concurrente se utilizaron las siguientes escalas:

- Cuestionario de habilidades de comunicación (CSQ).

- Escala de funcionamiento social (SFS).

- Escala sobre Perfil de habilidades de la vida cotidiana (LSP).

Para evaluar la validez discriminante se utilizaron:

- Escala de Funcionamiento (GAF).

- Escala de impresión clínica global (ICG).

\section{Procedimiento}

Se desarrolló un cuaderno de recogida de datos para garantizar la adecuada recogida de información y para mantener el mismo protocolo de exploración. Las personas que realizaron las entrevistas tenían formación específica y entrenamiento en la administración de los distintos instrumentos y eran los terapeutas referentes. Se realizó una selección aleatorizada de sujetos a partir del registro de casos de cada uno de los centros y se comprobó que cumplieran los criterios de inclusión en el estudio. Posteriormente se citó individualmente a cada usuario y se les informó del objetivo y contenido del mismo. Si finalmente aceptaban participar en el estudio, se procedía a la firma del documento de consentimiento informado, por parte del usuario/a y del investigador/a y se procedía a realizar la evaluación. Los datos se recogieron en los períodos comprendidos entre junio y diciembre de 2008. El proyecto fue evaluado por el Comité de Investigación y el Comité de Ética de Parc Sanitari Sant Joan de Déu y por el de la Fundació Althaia.

\section{Análisis Estadístico}

El diseño de la base de datos así como el análisis de éstos se realizó mediante el paquete estadístico SPSS/ PC+ (versión 19). Para el análisis de datos se utilizaron las siguientes pruebas: $\mathrm{El} \alpha$ de Cronbach se utilizó para determinar la consistencia interna del instrumento. La fiabilidad test-re-test fue evaluada a partir del coeficiente de correlación intraclases. Por último, se utilizó la Correlación de Pearson o de Spearman para valorar la convergente y la validez discriminante.

\section{Resultados}

Las características sociodemográficas de la muestra se presentan en la Tabla 1.

El alfa de Cronbach es elevada, de .936 en la escala total, de .935 en la subescala de grado de malestar y de .878 en la subescala de probabilidad de respuesta.
Tabla 1. Datos sociodemográficos de la muesta.

\begin{tabular}{ll}
\hline Variables & $\%(n)$ \\
\hline Sexo & \\
Hombres & $77.4 \%(96)$ \\
Mujeres & $22.6 \%(28)$ \\
Estado Civil & \\
Solteros/as & $88.8 \%(111)$ \\
Casados/as o Con pareja & $4.0 \%(5)$ \\
Separados/as o Divorciados/as & $6.4 \%(8)$ \\
Viudos/as & $.8 \%(1)$ \\
Núcleo de convivencia & \\
Solos/as & $11.2 \%(14)$ \\
Padres & $53.6 \%(67)$ \\
Otros familiares de primer grado & $4.8 \%(6)$ \\
Familia propia & $8.8 \%(11)$ \\
Dispositivos residencias de salud mental & $17.6 \%(22)$ \\
Centros psiquiátricos & $2.4 \%(3)$ \\
Pensiones & $1.6 \%(2)$ \\
Situación laboral & \\
Trabajo normalizado & $0.8 \%(1)$ \\
Trabajo protegido & $1.6 \%(2)$ \\
Paro contributivo & $2.4 \%(3)$ \\
Incapacidad laboral transitoria & $1.6 \%(2)$ \\
Amas de casa & $2.4 \%(3)$ \\
Jubilados/as o pensionistas & $84.8 \%(106)$ \\
Otros & $6.4 \%(8)$ \\
Edad & $\mathrm{Media}(S D)$ \\
& $42.43(10.29)$ \\
\hline
\end{tabular}

El coeficiente de correlación intraclase indica que la escala muestra un nivel aceptable de fiabilidad entre los dos momentos (Tabla 2). De las dos subescalas, en la escala de probabilidad de respuesta hay más ítems que presentan un grado de acuerdo bajo (inferior a .4). En los ítems 10 y 19 coincide un bajo acuerdo en las dos evaluaciones tanto en subescala de grado de malestar como en la de probabilidad de respuesta. Si eliminamos el ítem 10 y 19 el alfa de cronbach es de .931 en la subescala de grado de malestar y .871 en la de probabilidad de respuesta y .932 en el total de la escala.

En la muestra estudiada, hemos hallado que ningún ítem de la subescala del grado de malestar es superior a 3. Sin embargo, las medias de la probabilidad de respuesta de cada ítem son superiores a 3 en 21 ítems (Media=2.96). Esto quiere decir que la emisión de conductas en las situaciones planteadas es baja.

La Tabla 3 muestra los resultados de la validez convergente de la escala Gambrill y Richey con las escalas de habilidades sociales y subescalas concretas de instrumentos de funcionamiento social. La validez convergente de las dos subescalas de la Gambrill y Richey es muy elevada con la escala CSQ, tanto en el total como en las dos subescalas de las que está formada $(p<$.001). En relación a la escala SFS, la validez convergente es alta para la puntuación total y para las subescalas de aislamiento social y comunicación interpersonal $(p<.001-.05)$. La subescala de comunicación del LSP presenta una validez convergente con la escala validada, esta convergencia es alta $(p<.001-.024$ 
Tabla 2. Coeficiente de correlación intraclase en los dos momentos.

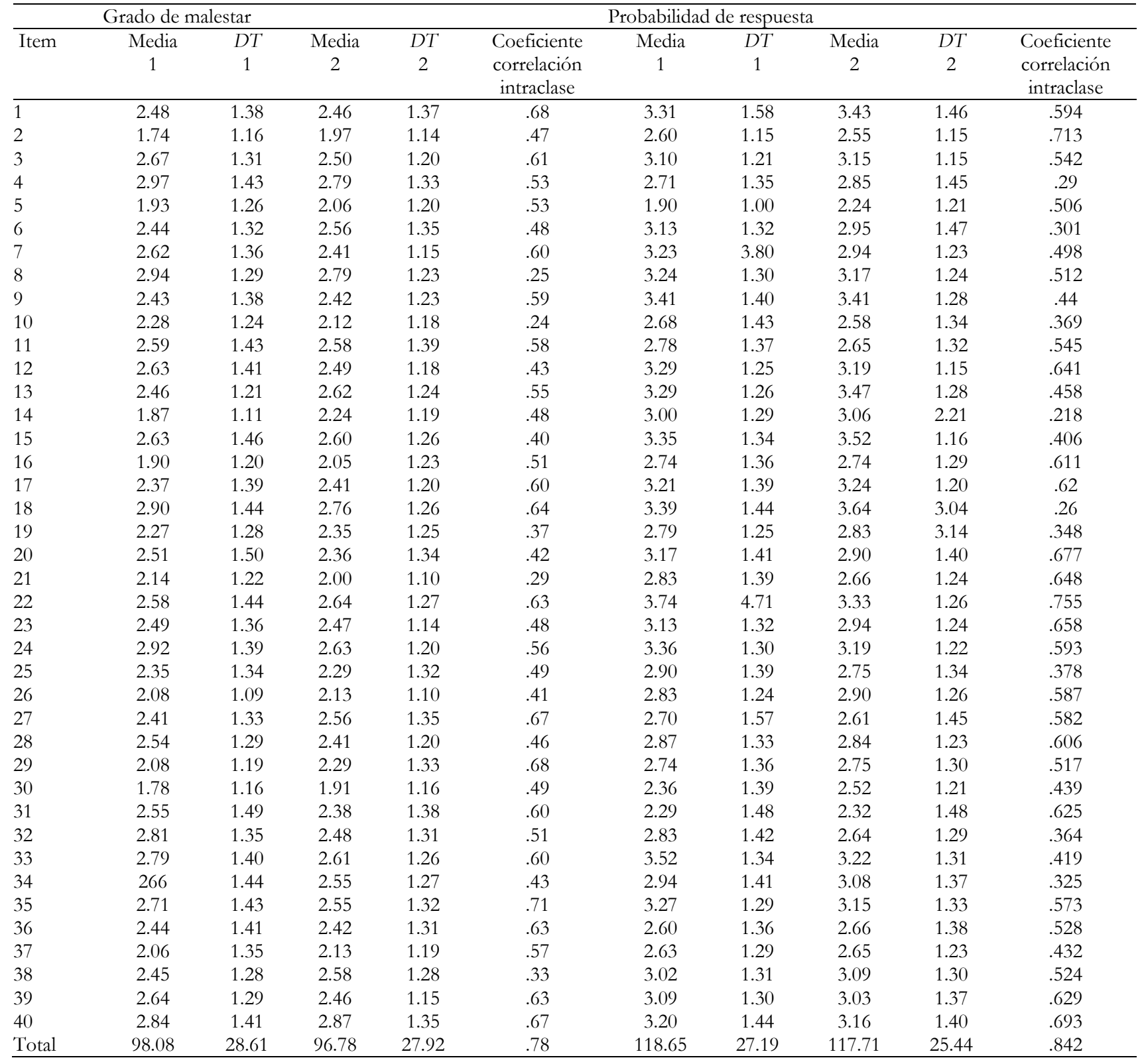

Tabla 3. Validez convergente de la escala de Gambrill y Richey con habilidades sociales y funcionamiento social.

\begin{tabular}{|c|c|c|c|c|c|c|}
\hline & Grado de malestar & $p$ valor & Probabilida de respuesta & $p$ valor & Puntuación total GR & $p$ valor \\
\hline EFS Aislamiento social & $-.206^{*}$ & .039 & $-.195^{*}$ & .050 & $-.234^{*}$ & .021 \\
\hline LSP Comunicación & $.208^{*}$ & .024 & $.300^{* *}$ & .001 & $.279^{* *}$ & .003 \\
\hline CSQ Habilidades de comunicación & $-.351^{* *}$ & .000 & $-.408^{* *}$ & .000 & $-.476^{* *}$ & .000 \\
\hline CSQ Habilidades personales & $-.454^{* *}$ & .000 & $-.436^{* *}$ & .000 & $-.553^{* *}$ & .000 \\
\hline CSQ Total & $-.500^{* *}$ & .000 & $-.481^{* *}$ & .000 & $-.610^{* *}$ & .000 \\
\hline
\end{tabular}

Los datos obtenidos en las distintas subescalas de la SFS y del LSP, son divergentes a excepción de aquellas que miden concretamente las relaciones sociales (aislamiento social y comunicación interpersonal del SFS y comunicación del LSP). En relación al GAF encontramos validez divergente con las subescalas de habilidades sociales de Gambrill y Ri- chey. En cuanto a la CGI encontramos validez divergente con probabilidad de respuesta, sin embargo, encontramos que hay validez convergente entre las subescalas de CGI y la subescala de grado de malestar de la Gambrill y Richey (Tabla 4). 
Tabla 4. Validez divergente de la escala de Gambrill y Richey con funcionamiento social y variables clínicas.

\begin{tabular}{|c|c|c|c|c|c|c|}
\hline & Grado de malestar & $p$ valor & Probabilidad de respuesta & $p$ valor & Puntuación total GR & $p$ valor \\
\hline EFS Independencia ejecución & -.131 & .166 & -.032 & .737 & -.101 & .302 \\
\hline EFS independencia competencia & -.060 & .537 & -.047 & .632 & -.063 & .530 \\
\hline EFS actividades del tiempo libre & .028 & .767 & -.067 & .477 & -.031 & .745 \\
\hline EFS conducta personal & -.069 & .478 & $-.219^{*}$ & .023 & -.146 & .141 \\
\hline EFS empleo & -.068 & .606 & -.242 & .072 & -.129 & .348 \\
\hline EFS Total & $-.337^{*}$ & .033 & $-.430^{* *}$ & .006 & $-.431^{* *}$ & .006 \\
\hline GAF social & -.172 & .060 & -.067 & .470 & -.129 & .172 \\
\hline GAF Clínico & .048 & .600 & .042 & .650 & .041 & .665 \\
\hline ICG Síntomas positivos & $.285^{* *}$ & .002 & .112 & .231 & $.234^{*}$ & .013 \\
\hline IGC síntomas negativos & $.197^{*}$ & .032 & .028 & .764 & .115 & .225 \\
\hline ICG síntomas depresivos & $.262^{* *}$ & .004 & .101 & .281 & $.192^{*}$ & .042 \\
\hline ICG síntomas cognitivos & $.192^{*}$ & .038 & .002 & .980 & .125 & .190 \\
\hline ICG Total & $.307^{* *}$ & .001 & .087 & .351 & $.236^{*}$ & .012 \\
\hline LSP Autocuidado & -.061 & .515 & .113 & .230 & .033 & .735 \\
\hline LSP Social interpersonal & -.034 & .721 & .095 & .317 & .032 & .738 \\
\hline LSP social no personal & -.104 & .268 & .051 & .589 & -.035 & .719 \\
\hline LSP vida autónoma & -.105 & .259 & -.107 & .253 & -.114 & .231 \\
\hline LSP total & -.166 & .081 & -.043 & .658 & -.114 & .248 \\
\hline
\end{tabular}

\section{Discusión}

Nuestros resultados muestran que el inventario de Gambrill y Richey presenta adecuadas propiedades psicométricas, tanto en cuanto a consistencia interna, validez convergente y divergente $y$, de fiabilidad temporal, en población con un diagnóstico de esquizofrenia. La muestra utilizada se caracteriza por encontrarse en procesos de rehabilitación y de reinserción comunitaria, en los cuales el manejo de las habilidades sociales es un indicador de pronóstico de evolución (Ahuir, Bernardo, de la Serna, Ochoa, Carlson, Escartin, GutierrezZotes, Valero, Salamero, Cañizares, Fernández-Egea, Cañete y Gallo, 2009). Por ello, la validación de un instrumento autoaplicado que mide asertividad, puede ser de gran utilidad para valorar fácilmente este aspecto de las habilidades sociales.

En la versión original, validada en población general, de la escala las puntuaciones medias de discomfort oscilaron alrededor de 95 (Gambrill y Richey, 1975). En nuestro estudio, la media de discomfort es ligeramente superior (aproximadamente dos puntos de diferencia), sin embargo, las puntuaciones obtenidas, no superan la media del instrumento (media $=3$ ). Este dato sugiere que las personas con esquizofrenia muestran un nivel de malestar ligeramente superior ante determinadas situaciones sociales, y que el grado de ansiedad, aunque no es excesivamente elevado, si es superior en las situaciones descritas en la escala. En relación a la subescala de probabilidad de respuesta, las puntuaciones obtenidas en nuestro trabajo presentan valores claramente inferiores a los hallados en la validación original, sugiriendo que las personas con esquizofrenia muestran un déficit en la probabilidad de emisión de conductas asertivas.

Ambos resultados conjuntamente indican que las personas con diagnóstico de esquizofrenia muestran una baja probabilidad de realizar determinadas conductas asertivas. Sin embargo, el grado de malestar obtenido no se corresponde con la baja probabilidad de respuesta (Gambrill y Richey,
1975). Estos datos nos indican que en la mayoría de personas con diagnóstico de esquizofrenia, no existe una anticipación de las situaciones que pueden generar malestar, posiblemente debido a los síntomas más característicos de la enfermedad, como es el caso del aplanamiento emocional (Viertiö, Tuulio-Henriksson, Perälä, Saarni, Koskinen, Sihvonen, Lönnqvist y Suvisaari, 2011) y los déficits en la planificación (Reichenberg, 2010). No obstante, nuestros datos sugieren fehacientemente que las personas con esquizofrenia tienen mayores dificultades en la gestión del estrés ante situaciones sociales, resultado que se halla de acuerdo con el perfil mayoritario, obtenido por otros autores, de las personas con esquizofrenia que se encuentran en proceso de rehabilitación (Phillips, Francey, Edwards y McMurray, 2009).

A nivel de fiabilidad, nuestro estudio ha determinado que en algunos casos el coeficiente de correlación intraclase es bajo (ítem 10 y 19). Estos resultados, al tratarse de un instrumento autoadministrado, pueden estar relacionados con las características de la población en la que se ha validado la escala; dado que las personas con diagnóstico de esquizofrenia muestran fluctuaciones sintomáticas y dificultades en la toma de decisiones (Dudley, John, Young y Over, 1997; Garety, Hemsley y Wessely, 1991; Moritz y Woodward, 2005) que pueden influenciar los resultados. Teniendo en cuenta este hecho, se podría plantear una revisión de estos ítems para la versión del inventario para personas con esquizofrenia, ya que al eliminar los ítems no se altera la consistencia interna del instrumento.

Un resultado no esperado en este trabajo es la relación positiva existente entre los diferentes tipos de sintomatología clínica, mediada mediante el ICG, y la subescala de grado de malestar del inventario de asertividad. Estos datos podrían explicarse por una posible relación entre la presencia de diferente tipo de sintomatología clínica, negativa y desorganizada $\mathrm{y}$, los déficits de funcionamiento social en las personas con esquizofrenia (Hunter y Barry, 2011; Vila-Rodríguez, Ochoa, 
Autonell, Usall y Haro, 2011). Por ello, podría ser esperable que las personas con mayor sintomatología también mostraran un mayor grado de malestar al realizar conductas asertivas.

La validación de la escala de asertividad de Gambrill y Richey que hemos realizado, tiene como limitación principal el tipo de población con la que se ha realizado el estudio, ya que nuestros resultados únicamente pueden generalizarse a personas con esquizofrenia que se hallen vinculados en dispositivos de rehabilitación. Por ello, serían necesarios más estudios a fin de poder validar este instrumento en personas con esquizofrenia que se encuentren en otros dispositivos y, en población con otros trastornos mentales graves. No obstante, los datos obtenidos sugieren que este instrumento es de utilidad para valorar la asertividad en personas con esqui-

\section{Referencias}

Anthony, W., Cohen, M. y Cohen, B. (1984). Psychiatric rehabilitación. En J.A. Talbott (ed.) The chronic mental patient. Five years later (pp.137-157). Orlando: Grune y Straton Inc.

Ahuir, M.; Bernardo, M., de la Serna, E., Ochoa, S., Carlson, J., Escartin, G., Gutierrez-Zotes, A., Valero, J., Salamero, M., Cañizares, S., FernándezEgea, E., Cañete, J. y Gallo, P. (2009). Adaptación y validación española de la Escala Pronóstica para la Esquizofrenia de Strauss y Carpenter. Revista de Psiquiatría y Salud Mental (Barc.), 2, 150-159

Birchwood, M., Smith, J., Cochrane, R., Wetton, S. y Copestake, S. (1990). The Social Functioning Scale. The development and validation of a new scale of social adjustment programmes with schizophrenic patients. British Journal of Psychiatry, 157, 853-859.

Bulbena, A., Burgés, V., Fernández, A., Autonell, J. y Melloni, F. (2007). Spanish adaptation and validation of the brief form of the Life Skills Profile-20: an instrument to assess daily living skills in real clinical settings. Actas Españolas de Psiquiatría, 35, 79-88.

Caballo, V.E. (1993). La multidimensionalidad conductual de las Habilidades Sociales: Propiedades psicométricas de una medida de autoinforme, la EMES-M. Psicología Conductual, 1, 221-231.

Carrasco I, Clemente, M. y Llavona, L. (1989). Análisis del Inventario de asertividad de Gambrill y Richey. Estudios de psicología, 37, 63-74.

Dudley, R.E., John, C.H., Young, A.N. y Over, D. E. (1997). The effect of self-referent material on the reasoning of people with delusions. British Journal of Clinical Psychology, 36, 575-584.

Endicott, J., Spitzer, R.L., Fleiss, J.L. y Cohen, J. (1976). The Global Assessment Scale. A procedure for measuring overall severity of psychiatric disturbance. Archives of General Psychiatry, 33, 766-771.

Farkas, M. y Anthony, W. (1982). The development of the rebabilitation model as a response to the shortcomings of the desinstitutinalization movement. Monografia $n^{\circ} 1$. Boston: Boston University.

Farkas, M., Anthony, W. y Cohen, M. (1989). Psychiatric rehabilitation: The approach and its programs. En M. Farkas y W. Anthony (eds.) Psychiatric rehabilitation programs. Putting theory into practice. (pp. 207-216). Baltimore y Londres: The Johns Hopkins University Press.

Study of the reliability, validity and internal consistency of the LSP scale (Life Skills Profile). Profile of activities of daily living. Actas Luso Españolas de Neurología, Psiquiatría y Ciencias Afines, 20,71-5.

Gambrill, E. y Richey, C. (1975). An Assertion Inventory for Use in Assessment and Research. Behavior Therapy, 6, 550-561.

Garety, P.A., Hemsley, D.R. y Wessely, S. (1991). Reasoning in deluded schizophrenic and paranoid patients. Biases in performance on a probabilistic inference task. Journal of Nervous and Mental Diseases, 179, 194 201.

Green, M.F., Kem, R.S., Braff, D.L. y Mintz, J. (2000). Neurocognitive Deficits and functional autcome in schizophrenia: are we measuring the “right stuff'?. Schizopbrenia Bulletin, 26, 119-36. zofrenia, hecho que puede orientar la rehabilitación de la gestión de habilidades sociales, elemento clave que pronostica una mejor evolución de la enfermedad (Mueser, Kosmidis y Sayers, 1992). De hecho, es el primer inventario autoadministrado de asertividad que se ha validado en esta población.

La validación del inventario de asertividad de Gambrill y Richey en población con esquizofrenia, permite disponer de un instrumento de fácil administración y corrección, que proporciona una rápida información sobre el nivel de asertividad; hecho que permite orientar la intervención rehabilitadora en este ámbito.

Agradecimientos.- Beca de Investigación del Parc Sanitari Sant Joan de Déu (2010).

Haro, J.M., Kamath, S.A., Ochoa, S., Novick, D., Rele, K., Fargas, A., Rodriguez, M.J., Rele, R., Orta, J., Kharbeng, A., Araya, S., Gervin, M., Alonso, J., Maureas, V., Lavrentzou, E., Liontos, N., Grefor, K., Jones, P.B., SOHO Study Group. (2003). The Clinical Global ImpressionSchizophrenia Scale: a simple instrument to measure the diversity of symptoms present in schizophrenia. Acta Psychiatrica Scandinavica, Suppl. 416, 16-23.

Heissen, R.K., Liberman, R.P. y Kopelowicz, A. (2000). Psychosoical skills training for schizophrenia: lessons from the laboratory. Schizophrenia Bulletin, 26, 21-46.

Hunter, R. y Barry S. Negative symptoms and psychosocial functioning in schizophrenia: Neglected but important targets for treatment. European Psychiatry. Doi: 10.1016/j.eurpsy.2011.02.015.

Labrador, F.J.; Cruzado, J.A. y Muñoz, M. (2004). Manual de técnicas de modificación y terapia de conducta. Madrid: Pirámide.

Liberman R.P. (1995). Rehabilitación Integral del Enfermo Mental Crónico. Barcelona: Martínez Roca (Orig. 1988).

Liberman, R.P. y Kopelowicz A. (2004). Un enfoque empírico de la recuperación de la esquizofrenia: definir la recuperación e identificar los factores que pueden facilitarla. Rehabilitación psicosocial, 1, 12-29.

Liberman, R.P., Wallace, Ch., Blackwell, G., Eckman, T.A., Baccaro, J.V. y Kuenhel, T.G. (1993). Innovations in skills for the seriously mentally ill: The UCLA social and independent living skills modules. Innovations and Research, 2, 43-60.

Moritz, S. y Woodward, T.S. (2005). Jumping to conclusions in delusional and non-delusional schizophrenic patients. British Journal of Clinical Psychology, 44, 193-207.

Mueser, K.T., Kosmidis, M.H. y Sayers, M.D. (1992). Symptomatology and the prediction of social skills acquisition in schizophrenia. Schizophrenia Research, 8, 59-68.

Olivares, J.M. y Torres, A. (2005). Validación en castellano de la Social Functioning Scale. Actas Españolas de Psiquiatría, 333, 216-220.

Orviz, S. y Fernández, J.A. (2002). Entrenamiento en habilidades sociales para personas con problemas psiquiátricos crónicos. En A. Rodríguez (Coord.), Rebabilitación psicosocial de personas con trastornos mentales crónicos. (pp:229-247). Madrid: Pirámide.

Otero, V. (2004). Rebabilitación de enfermos mentales crónicos. Evaluación de objetivos. Barcelona: Ars Medica.

Prat, G., Casas-Anguera, E., Garcia-Franco, M., Escandell, MJ., Martín, JR., Vilamala, S., Villalta- Gil, V., Gimenez-Salinas, J., Hernandez-Rambla, C., Ochoa, S. (2009, november). Validation study for Spanish subjects with a diagnosis of schizophrenia of the "Communication Skills Questionnaire. Póster presentado en el $15^{\text {th }}$ Biennial Winter Workshop in Psychoses, Barcelona, España.

Phillips,L.J., Francey, S.M., Edwards, J. y McMurray, N. (2009). Strategies used by psychotic individuals to cope with life stress and symptoms of illness: a systematic review. Anxiety and Stress Coping, 22, 371-410. 
Raich, R., Escursell, M. y Vila-Vidal, J.R. (1985). Estudi de l'inventari d'assertivitat de Gambrill i Richey en una mostra d'estudiants de Psicologia de la UAB. Quaderns de Psicologia, I, 127-141.

Reichenberg, A. (2010). The assessment of neuropsychological functioning in schizophrenia. Dialogues in Clinical Neurosciences, 12, 383-92.

Rosen, A., Hadzi-Paulovic, D. y Porter, G. (1989). The life skills profile: a measure assessing function and disability in schizophrenia. Schizophrenia Bulletin, 15, 325-337.

Takahashi, M., Tanaka, K. y Miyaoka, H. (2006). Rehability and validity of communication skills questionnaire (CSQ). Psychiatry and Clinical Neurosciences, 60, 211-218.
Viertiö, S., Tuulio-Henriksson, A., Perälä, J., Saarni, S.I., Koskinen, S., Sihvonen, M., Lönnqvist, J. y Suvisaari, J. .Activities of daily living, social functioning and their determinants in persons with psychotic disorder. European Psychiatry. Doi: 10.1016/j.eurpsy.2010.12.005.

Vila-Rodriguez, F., Ochoa, S., Autonell, J., Usall, J. y Haro, J.M. (2011) Complex Interaction Between Symptoms, Social Factors, and Gender in Social Functioning in a Community-Dwelling Sample of Schizophrenia. Psychiatric Quarterly, 82, 261-274..

Wallace, C. y Liberman, R. (1985). Social skills training for patients with schizophrenia: a controlled clinical trial. Psychiatry Research, 15, 239-47.

(Artículo recibido: 11-6-2012; revisado: 19-11-2012; aceptado: 22-2-2013) 